\title{
88. On the Asymptotic Distribution of the Sum of Independent Random Variables.
}

By Shigeru TAKahashi.

(Comm. by Z. Suetuna, M.J.A., Oct. 12, 1951.)

$\S 1$. Let $\left\{X_{i}\right\} \quad i=1,2, \ldots$ be a sequence of independent random variables defined in a probability space $(\Omega, F, P)$. The so-called central limit theorem" states that when a sequence $\left\{X_{i}\right\}$ satisfies certain conditions then

$$
\lim _{n \rightarrow \infty} P\left(\sqrt{\frac{1}{n}} \sum_{i=1}^{n} X_{i}(\omega) \leqq a\right)=\frac{1}{\sqrt{2 \pi}} \int_{-\infty}^{n} e^{-u^{2} / 2} d u=G(a),
$$

where $\sqrt{\frac{1}{n}} \sum_{i=1}^{n} X_{i}$ denotes suitably normalized variable. Concerning this theorem we consider following two generalizations:

$1^{\circ}$ Replace a constant upper limit a of summation by a measurable function $g(\omega)$ defined in $\Omega$.

$2^{\circ}$ Replace the number $n$ of random variables of summation by a random function $N_{n}(\omega)$ defined in $\Omega$.

On these generalizations many theorems have been proved ${ }^{\text {3). }}$. Let $\left\{X_{i}\right\}$ be a sequence of independent random variables satisfying the central limit theorem (I). For any real numbers $a$ and $b$, we define the sets $E_{a, b}^{i}=\left[\omega ; a \leqq X_{i}(\omega)<b\right]$ and denote by $\bar{F}^{\prime}$ the smallest Borel field which includes all the sets $E_{a, b}^{i}$ defined for any $a, b$ and $i=1,2, \ldots$ We complete $\bar{F}$ with respect to the measure $P$ and denote it by $\bar{F}$. In $\$ 3$ we prove the following:

Theorem 1. If $E \varepsilon \bar{F}$, then

$$
\lim _{n \rightarrow \rightarrow \infty} P\left(\sqrt{\sqrt{n}} \sum_{i=1}^{n} X_{i}(\omega) \leqq a, E\right)=P(E) G(a) .
$$

In order to prove this theorem we show some lemmas in $\$ 2$, and in $\$ 4$ we consider the above generalizations by using Theorem I.

To define and to discuss the problems on $\left\{X_{i}\right\}$, it is sufficient to consider the probability space $(\Omega, F, P)$ as $(\Omega, \tilde{F}, P)$. So the theorems proved in $\$ 4$ give the answer of the above generalizations for independent sequence.

$\S 2$. First of all we consider a sequence $\left\{X_{i}\right\}$ which satisfies following conditions :

1) H. Cranier, Random variable and its probability distribution. Cambridge (1937).

2) J. C. Smith, On the asymptotic distribution of the sum of Rademacher functions. Bull. Amer. Math. Soc., vol. 51 (1945).

H. Robbins, On the sum of random number of random variables. Bull. Amer. Math. Soc., vol. 54 (1948).

S. Takahashi, On the central limit theorem (under the press). 
$1^{\circ} .\left\{X_{i}\right\}$ is an independent sequence.

$2^{\circ}$. $\left\{X_{i}\right\}$ satisfies the central limit theorem (I).

$3^{\circ}$. For each $i$, the set of values which $X_{i}$ takes is at most enumerable.

Let $a_{i s}^{i}$ be the values which $X_{i}$ takes. Put $P_{s}^{i}=P\left[X_{i}=a_{n}^{i}\right]$, $A_{k}^{i}=\left[\omega ; X_{i}=a_{k}^{i}\right]$ and $F^{\circ}$ the smallest Borel field which includes all the sets $A_{k}^{i} k=1,2, \ldots, i=1,2, \ldots$. We assume that' $P_{k}^{i}$ is a non-increasing sequence of $k$ for each $i$. Then $\left(\Omega, F^{\circ}, P\right)$ is also a probability space. For any sequence (finite or infinite) of integers $i_{1}, i_{2}, \ldots, i_{n}$ we define the set

$$
A_{i 1}, i_{i 2}, \ldots \ldots, i_{i_{2}}=\bigcap_{i=1}^{n} A_{i_{l}}^{\iota} \text {. }
$$

Then, from the independency of $\left\{X_{i}\right\}$

$$
\begin{gathered}
P\left(A_{i_{1}}, i_{2}, \ldots, i_{n}\right)=P\left(\bigcap_{l=1}^{n} A_{i_{l}}^{l}\right)=P\left(\bigcap_{l=1}^{n} X_{l}=a_{i_{l}}^{l}\right) \\
=\prod_{l=1}^{n} P\left(X_{l}=a_{i_{l}}^{l}\right)=\prod_{l=1}^{n} P_{i_{l}}^{l}
\end{gathered}
$$

If for some infinite sequence $i_{1}, i_{2}, \ldots, i_{n}, \ldots, P\left(A_{i_{1}}, i_{2}, \ldots, i_{n}, \ldots\right)$ $=P>0$, then $\lim _{n \rightarrow \infty} P\left(\sqrt{\frac{1}{n}} \sum_{i=1}^{n} X_{i}=\frac{1}{\sqrt{n}} \sum_{i=1}^{n} a_{i_{l}}^{l}\right)=P$. This contradicts with the assumption $2^{\circ}$.

Hence for any infinite sequence $i_{1}, i_{2}, \ldots, i_{m}, \ldots$.

$$
P\left(A_{i_{1}}, i_{2}, \ldots, i_{i_{n}}, \ldots\right)=0 \text {. }
$$

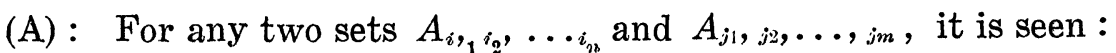

$1^{\circ}$. If there exists at least one $k$ such that $k \leqq m, n$ and $i_{k} \neq j_{k}$, then $A_{i_{1}}, i_{2}, \ldots, i_{n} \cap A_{j_{1}}, j_{2}, \ldots, j_{m}=\Theta$, where $\Theta$ denotes the empty set.

$2^{\circ}$. If $m=n$ and $i_{k}=j_{k}$ for all $k \leqq m=n$, then

$$
A_{i_{1}}, i_{2}, \ldots, i_{i_{2}}=A_{j_{1}, i_{2}}, \ldots,,_{j_{m}} \text {. }
$$

$3^{\circ}$. If $m=n$ and $i_{k}=j_{k}$ for all $k \leqq m$, then

$$
A_{i_{1}}, i_{2}, \ldots,,_{i_{n}}<A_{j_{1}}, j_{2}, \ldots,,_{j_{m}} \text {. }
$$

Next we define a probability space $(T, B, m)$ as follows :

$1^{\circ}$. $T$ is the interval $[0.1>$.

$2^{\circ}$. $B$ is the class of $B$-measurable sets in $[0,1>$.

$3^{\circ}$. $m$ is Lebesgue measure.

In $T$ we define the set $E_{i_{1}}, i_{2}, \ldots, i_{n}$ for any sequence of integers ${ }_{i_{1}}, i_{2}, \ldots, i_{n}$.

$1^{\circ} . \quad E_{i_{\mathbf{1}}}=\left[t: \sum_{K=1}^{i_{1}-1} P_{K}^{1} \leqq t<\sum_{K=1}^{i_{1}} P_{K}^{1}\right]$.

$2^{\circ}$. If $E_{i_{1}}, i_{2}, \ldots, i_{n^{-1}-1}$ has been defined and the interval $\left[d_{1}, d_{2}>\right.$ denotes then

$$
E_{i_{1}}, i_{2}, \ldots, i_{n}=\left[t ; d_{1}+\left(d_{2}-d_{1}\right) \sum_{k=1}^{i_{n-1}} P_{k} \leqq t<d_{1}+\left(d_{2}-d_{1}\right) \sum_{k=1}^{i_{n}} P_{k}^{i}\right]
$$


From the above construction and (II)

$$
m\left(E_{i_{1}}, i_{2}, \ldots, i_{n}\right)=I_{l=1}^{n} P_{i_{l}}^{l}=P\left(A_{i_{1}}, i_{2}, \ldots, i_{i_{2}}\right) \text { for all } i_{1}, i_{2}, \ldots, i_{n} . \text { (IV) }
$$

For any infinite sequence $i_{1}, i_{2}, \ldots, i_{n 2}, \ldots$

$$
m\left(E_{i_{1}}, i_{2}, \ldots, i_{n}, \ldots\right)=0 \text {. }
$$

By (V), it is seen that the smallest Borel field which includes all the sets $E_{i_{1} i_{2}} \ldots i_{n}$ is identical with the class $B$.

(B): For any two sets $E_{i_{1}}, i_{2}, \ldots, i_{n}$ and $E_{j_{1}}, i_{2}, \ldots, j_{m}$, it is seen :

$1^{\circ}$. If there exists at least one $k$ such that $i_{k} \neq j_{k}, k \leqq m, n$, then

$$
E_{i_{1}}, i_{2}, \ldots, i_{i_{2}} \cap E_{j_{1}}, i_{2}, \ldots, j_{m}=\Theta .
$$

$2^{\circ}$. If $m=n$ and $i_{k}=j_{k}$ for all $k \leqq m=n$, then

$$
E_{i_{1}}, i_{2}, \ldots, i_{i_{i}}=E_{j_{1}, j_{2}}, \ldots, j_{m} \text {. }
$$

$3^{\circ}$. If $m<n$ and $i_{k}=j_{k}$ for all $k \leqq m$, then

$$
E_{i}, i_{2}, \ldots, i_{i_{n}}<\quad E_{j_{1}, j_{2}}, \ldots,,_{i_{m}} \text {. }
$$

By $(A)$ and $(B)$ we can define a transformation $\phi$ from $B$ to $F^{\circ}$ as follows :

$1^{\circ} . \phi(\Theta)=\Theta$.

$2^{\circ} . \varphi\left(E_{i_{1}}, i_{2}, \ldots,{ }_{i_{22}}\right)=A_{i_{1}}, i_{2}, \ldots,{ }_{i_{22}}$ for all $i_{1}, i_{\mathrm{a}}, \ldots i_{22}$.

$3^{\circ} . \varphi\left(\bigcup_{i=1}^{\infty} E_{i}\right)=\bigcup_{i=1}^{\infty} \varphi\left(E_{i}\right), \quad E_{i} \varepsilon B$.

From $1^{\circ}-3^{\circ}$ it follows that

$4^{\circ} . \varphi(T)=\Omega$ and $\varphi(E)=\varphi(T)-\varphi\left(E^{\prime}\right)$ where $E^{\prime \prime}$ denotes the complement of $E$.

$$
5^{\circ} . \phi\left(\bigcap_{i=1}^{\infty} E\right)=\bigcap_{i=1}^{\infty} \varphi\left(E_{i}\right), \quad E_{i}^{\prime} \varepsilon B .
$$

Hence $\phi$ is a one to one correspondence between the sets of $B$ and sets of $F^{2}$.

By (IV) and the properties of $\varphi$, it follows that;

$1^{\circ}$. if $E \& B$ then $\phi(E) \varepsilon F^{\circ}$ and $m(E)=P(\varphi(E))$,

$2^{\circ}$. if $E \varepsilon F^{\circ}$ then $\phi^{-1}(E) \varepsilon B$ and $m\left(\phi^{-1}(E)\right)=P(E)$.

We define a sequence of random variables $\left\{Y_{i}(t)\right\} i=1,2 \ldots$ as follows, if $t \varepsilon E_{k_{1}, k_{2}}, \ldots, k_{i}$ then $Y_{i}(t)=a_{k_{i}}^{i}$ for all $k_{1}, k_{2}, \ldots, k_{i}, i=1,2, \ldots$.

Thus defined sequence $\left\{Y_{i}(t)\right\}$ is independent. For, $m\left(\bigcap_{i=1}^{n} Y_{i}(t)\right.$ $\left.=a_{k_{i}}^{i}\right)=m\left(E_{k_{1}}, k_{2}, \ldots, k_{k_{3}}\right)=\prod_{b=1}^{n} P_{k_{l}}^{l}$. On the other hand

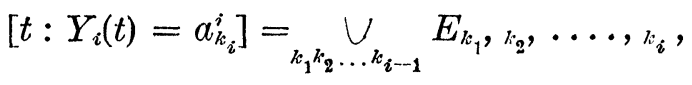

where $\bigcup_{k_{1} k_{2} \ldots k_{i-1}}$ denotes the summation for all possible combinations of $k_{1}, k_{2}, \ldots, \ddot{k}_{i-1}$. According to $(B)$ and (IV)

$$
\begin{aligned}
m\left[t ; Y_{i}(t)\right. & \left.=a_{k_{i}}^{i}\right]=\sum_{k_{1} k_{2} \ldots k_{k_{i}-1}} m\left(E_{k_{1}}, k_{2}, \ldots, k_{i-1}, k_{i}\right) \\
& =\sum_{k_{1} k_{2} \ldots k_{i-4}} P_{k_{l}}^{b 2-1} \prod_{b=1}^{n-1} P_{i_{i}}^{n}=P_{k_{i}}^{i} .
\end{aligned}
$$


Hence $m\left(\bigcap_{i=1}^{n} Y_{i}(t)=\alpha_{k_{i}}^{i}\right)=\prod_{i=1}^{n} m\left(Y_{i}(t)=a_{k_{i}}^{i}\right)$ for all $n$. By the fact that $Y_{i}(t)$ takes on $E_{k_{1}}, k_{2}, \ldots,{ }_{k_{i}}$ the same value as that $X_{i}$ takes on $A_{k_{1}}, k_{2}, \ldots, k_{i}$ and by the properties of $\varphi$ it is seen for all $n$ that

Hence $\quad m\left(\frac{1}{\sqrt{n}} \sum_{i=1}^{n} Y_{i}(t) \leqq a\right)=P\left(\sqrt{\frac{1}{n}} \sum_{i=1}^{n} X_{i}(\omega) \leqq a\right)$,

so

$$
\begin{aligned}
\lim _{n \rightarrow \infty} m\left(\sqrt{ } \frac{1}{n} \sum_{i=1}^{n} Y_{i}(t) \leqq a\right) & =\lim _{n \rightarrow \infty} P\left(\sqrt{\frac{1}{n}} \sum_{i=1}^{n} X_{i}(\omega) \leqq a\right) . \\
& =G(a) .
\end{aligned}
$$

Lemma l. If $E \varepsilon B$, then

$$
\lim _{n \rightarrow \infty} m\left(\sqrt{\frac{1}{n}} \sum_{i=1}^{n} Y_{i}(t) \leqq a, E\right)=m(E) G(a) \text {. }
$$

Proof. For any finite sequence of integers $i_{1}, i_{2}, \ldots, i_{l}$

$$
\begin{aligned}
& \lim _{n \rightarrow \infty} m\left(\sqrt{\frac{1}{n}} \sum_{i=1}^{n} Y_{i}(t) \leqq a, E_{i_{1}}, \ldots, i_{i_{2}}, \ldots\right. \\
& =\lim _{n \rightarrow \infty} m\left(\sqrt{\frac{1}{n}}\left(\sum_{i=1}^{b}+\sum_{i=l+1}^{n}\right) Y_{i}(t) \leqq a, E_{i_{1}}, i_{2}, \ldots, i_{l}\right) \\
& =\lim _{n \rightarrow \infty} m\left(\sqrt{ }, \frac{1}{n} \sum_{i=l+1}^{n} Y_{i}(t) \leqq a, E_{i_{1}}, i_{2} \ldots, i_{i}\right) \\
& =\lim _{n \rightarrow \infty} m\left(\sqrt{\frac{1}{n}} \sum_{i=b+1}^{n} Y_{i}(t) \leqq a, \bigcap_{k=1}^{l} X_{k}=a_{i_{k}}^{l}\right) \\
& =\lim _{n \rightarrow \infty} m\left(\sqrt{\frac{1}{n}} \sum_{i=b+1}^{n} Y_{i}(t) \leqq a\right) m\left(\bigcap_{k=1}^{l} X_{k}=a_{i_{k}}^{k}\right) \\
& =\lim _{n \rightarrow \infty} m\left(\sqrt{ } \frac{1}{n} \sum_{i=1}^{n} Y_{i}(t) \leqq a\right) m\left(E_{i_{1}}, i_{2}, \ldots, i_{i}\right)=G(\alpha) m\left(E_{i_{1}}, i_{2}, \ldots, i_{i}\right) .
\end{aligned}
$$

Now let $M$ denote the family of sets $E$ which satisfy the following relation

$$
\lim _{n \rightarrow \infty} m\left(\sqrt{\frac{1}{n}} \sum_{i=1}^{n} Y_{i}(t) \leqq a, E\right)=m(E) G(a) .
$$

Then, about $M$ it is seen that:

$1^{\circ}$. $M$ includes $E_{i_{1}}, i_{2}, \ldots, i_{i}$ for all ${ }_{i_{1}}, i_{2}, \ldots, i_{i}{ }^{l}(l=1,2, \ldots)$. For a finite sequence $i_{1}, i_{2}, \ldots, i_{l}$ we have proved above, but for infinite sequence it is evident from (V).

$2^{\circ}$. If $E<E^{\prime}$ and $E, E^{\prime} \varepsilon M$, then $E^{\prime}-E \varepsilon M$.

For, $\lim _{n \rightarrow \infty} m\left(\sqrt{ } \frac{1}{n} \sum_{i=1}^{n} Y_{i}(t) \leqq a, E^{\prime}-E\right)$

$=\lim _{n \rightarrow \infty} m\left(\sqrt{\frac{1}{n}} \sum_{i=1}^{n} Y_{i}(t) \leqq a, E^{\prime}\right)-\lim _{n \rightarrow \infty} m\left(\sqrt{\frac{1}{n}} \sum_{i=1}^{n} Y_{i}(t) \leqq a, E\right)$

$=G(a) m\left(E^{\prime}\right)-G(a) m(E)=G(a) m\left(E^{\prime}-E\right)$. 
$3^{\circ}$. If $E=\bigcup_{j=1}^{\infty} \Delta_{j}, \Delta_{j} \varepsilon M$ and $\Delta_{j}, \Delta_{j^{\prime}}$ are non-overlapping $\left(j \neq j^{\prime}\right)$, then $E \& M$.

$$
\text { For, } \begin{aligned}
\lim _{n \rightarrow \infty} m\left(\sqrt{\frac{1}{n}} \sum_{i=1}^{n} Y_{i}(t) \leqq a, E\right) \\
=\lim _{n \rightarrow \infty} \sum_{j=1}^{n} m\left(\frac{1}{\sqrt{n}} \sum_{i=1}^{n} Y_{i}(t) \leqq \alpha, E\right) .
\end{aligned}
$$

For all $n, \quad 0 \leqq m\left(\sqrt{\frac{1}{n}} \sum_{i=1}^{n} Y_{i}(t) \leqq a, \quad \Delta_{j}\right) \leqq m\left(\mathcal{d}_{j}\right)$

and

$$
\sum_{j=1}^{\infty} m\left(\mathcal{J}_{j}\right)=m(E) \text {. }
$$

Hence the convergence of $\sum_{j=1}^{\infty} m\left(\sqrt{\frac{1}{n}} \sum_{i=1}^{n} Y_{i}(t) \leqq a, \Delta_{j}\right)$ is unifom with respect to $n$. So we can exchange the order of $\lim$ and $\Sigma$ of (VI). We have therefore

$$
(\mathrm{VI})=\sum_{j=1}^{\infty} \lim _{n \rightarrow \infty} m\left(\sqrt{\frac{1}{n}} \sum_{i=1}^{n} Y_{i}(t) \leqq a, \Delta_{j}\right)=\sum_{j=1}^{8} G(a) m\left(\Delta_{j}\right)=G(a) m(E) .
$$

By $1^{\circ}-3^{\circ}$ and the fact that $m$ denotes Lebesgue measure, it follows that $M$ includes all sets of $B$.

We complete $F^{\circ}$ with respect to the measure $P$ and denote it by $\bar{F}^{\prime}$.

Lemma 2. If $E \varepsilon F^{\prime}$, then

$$
\lim _{n \rightarrow \infty} P\left(\frac{1}{v^{\prime}} \sum_{i=1}^{n} X_{i}(\omega) \leqq a, E\right)=P(E) G(a)
$$

Proof: It is sufficient to prove this lemma for the case $E \varepsilon F^{\circ}$. For all $n, \phi^{-1}\left(\sqrt{\sqrt{ }} \frac{1}{n} \sum_{i=1}^{n} X_{i}(\omega) \leqq a, E\right)=\left(\stackrel{1}{V^{\prime}} \sum_{i=1}^{n} Y_{i}(t) \leqq a, \phi^{-1}(E)\right)$. Hence $\quad \lim _{n \rightarrow \infty} P\left(\frac{1}{\eta} \sum_{i=1}^{n} X_{i}(\omega) \leqq a, E\right)$

$$
\begin{aligned}
& =\lim _{n \rightarrow \infty} m\left(\sqrt{\frac{1}{n}} \sum_{i=1}^{n} Y_{i}(t) \leqq a, \phi^{-1}(E)\right) \\
& =G(a) m\left(\phi^{r-1}(E)\right)=G(a) P(E) .
\end{aligned}
$$

$\S 3$. In this paragraph, we prove the theorem mentioned in $\S 1$. Let $\left\{X_{i}\right\}$ be a sequence of independent random variables satisfying the central limit theorem (I).

Now, let $\left(h_{i, k}\right)(i=1,2 \ldots, k=0, \pm 1, \pm 2, \ldots)$ be a sequence of real numbers satisfying the following conditions :

$1^{\circ}$. $h_{i},{ }_{k}>0$ for $k>0, h_{i},{ }_{k}<0$ for $k<0$. $h_{i, k}=0$ for $k=0$.

$2^{\circ}$. $\left(h_{i},,_{k}\right) \leqq h_{i}$ for all $k$ and $\sum_{i=1}^{n} h_{i}=O(\sqrt{n})(n \rightarrow \infty)$.

$3^{\circ} \cdot \sum_{k=0}^{\infty} h_{i}, k=+\infty \quad \sum_{k=0}^{\infty} h_{i, k}=-\infty$ for each $i$.

Using this sequence $\left(h_{i}, k_{k}\right)$, we define a sequence of random vari- 
ables $\left\{Z_{i}(\omega)\right\}(i=1,2, \ldots)$ as follows :

$$
\begin{aligned}
& Z_{i}(\omega)=\sum_{i=0}^{n} h_{i},{ }_{k} \text { if } \omega \varepsilon E_{i},{ }_{n}=\left[\omega ; \sum_{i=0}^{n} h_{i},{ }_{k} \leqq X_{i}(\omega)<\sum_{i=0}^{n+1} h_{i},{ }_{k}\right] \\
& \quad \text { for } n=0, \pm 1, \pm 2, \ldots, i=1,2, \ldots .
\end{aligned}
$$

Thus defined sequence $\left\{Z_{i}(\omega)\right\} i=1,2, \ldots$ is independent.

For, $P\left(\bigcap_{i=1}^{n} Z_{i}(\omega)=\sum_{k=0}^{n_{i}} h_{i}, k\right)=P\left(\bigcap_{i=1}^{n}\left[\sum_{i=0}^{n_{i}} h_{i},,_{k} \leqq X_{i}(\omega)<\sum_{i=0}^{n_{i}+1} h_{i}, k\right)\right.$

$$
=I_{i=1}^{n} P\left[\sum_{k=0}^{n_{i}} h_{i},{ }_{k} \leqq X_{i}(\omega)<\sum_{k=0}^{n^{2}+1} h_{h},{ }_{k}\right]=I_{i=1}^{n} P\left(Z_{i}(\omega)=\sum_{i=0}^{n_{i}} h_{i},{ }_{k}\right) .
$$

$\left\{Z_{i}(\omega)\right\}$ satisfies the central limit theorem (I).

For,

$$
P\left(\left|\sum_{i=1}^{n}\left(X_{i}-Z_{i}\right) / \sqrt{n}\right| \leqq \varepsilon\right) \geqq P\left(\sum_{i=1}^{n} h_{i} / \sqrt{n}<\varepsilon\right)
$$

for all $n$. On the other hand $\sum_{i=1}^{n} h_{i}=o(\sqrt{n})$. Hence

$$
\lim _{n \rightarrow \infty} P\left(\left|\sum_{i=1}^{n}\left(X_{i}-X_{i}\right) / \sqrt{n}\right|>\varepsilon\right) \geqq \lim _{n \rightarrow \infty} P\left(\sum_{i=1}^{n} h / \sqrt{n}<\varepsilon\right)=1 .
$$

So

$$
\sqrt{\frac{1}{n}} \sum_{i=1}^{n}\left(X_{i}-Z_{i}\right) \rightarrow 0(n \rightarrow \infty) \text { in probability. }
$$

Therefore $\lim _{n \rightarrow \infty} P\left(\sqrt{\frac{1}{n}} \sum_{i=1}^{n} Z_{i}(\omega) \leqq a\right)$

$$
\begin{aligned}
& =\lim _{n \rightarrow \infty} P\left(\frac{1}{\sqrt{n}} \sum_{i=1}^{n}\left(Z_{i}(\omega)-X_{i}(\omega)+X_{i}(\omega)\right) \leqq a\right) \\
& =\lim _{n \rightarrow \infty} P\left(\sqrt{\frac{1}{n}} \sum_{i=1}^{n} X_{i}(\omega) \leqq a\right)=G(a) .
\end{aligned}
$$

Now, let $F^{*}$ be the smallest Borel field which includes $E_{i, n}$ for $n=0, \pm 1, \pm 2, \ldots, \quad i=1,2, \ldots$ Then by Lemma $2 E \varepsilon F^{*}$, and

$$
\lim _{n \rightarrow \infty} P\left(\sqrt{\frac{1}{n}} \sum_{i=1}^{n} Z_{i}(\omega) \leqq \alpha, E\right)=P(E) G(a) \text {. }
$$

By (VIII) and (VII), if $E \varepsilon F^{*}$,

$$
\lim _{n \rightarrow \infty} P\left(\sqrt{\frac{1}{n}} \sum_{i=1}^{n} X_{i}(\omega) \leqq a, E\right)=P(E) G(a) \text {. }
$$

Next, we state Theorem 1 by using the definitions of $\bar{F}$ and $\tilde{F}$ mentioned in $\S 1$.

Theorem 1. If $E \varepsilon \tilde{F}$, then

$$
\lim _{n \rightarrow \infty} P\left(\sqrt{\frac{1}{n}} \sum_{i=1}^{n} X_{i}(\omega) \leqq a, E\right)=P(E) G(a) \text {. }
$$

Proof. It is sufficient to prove this theorem for the case where $E \varepsilon \bar{F}$. To define any set $E$ belonging to $\overline{F^{\prime}}$, it is sufficient to consider at most enumerable sets of the type of $\left[\omega ; a \leqq X_{i}(\omega)<b\right]$ for each $i$. So we can choose $\left(h_{i}, r_{i}\right)$ such that the set $E$ belongs to $F^{*}$ determined by $\left(h_{i},,_{k}\right)$. From (IX), Theorem 1 holds for this set.

$\$ 4$ Theorem 2. Let $g(\omega)$ be a non-negative $\tilde{F}$-measurable function. Then 


$$
\lim _{n \rightarrow \infty} P\left(\sqrt{\frac{1}{n}}\left|\sum_{i=1}^{n} X_{i}(\omega)\right| \leqq g(\omega)\right)=\frac{1}{\sqrt{2 \pi}} \int_{\Omega} P(d \omega) \int_{-g(\omega)}^{g(\omega)} e^{-u u^{2} / 2} d u
$$

Proof. It is sufficient to prove this theorem for the case where $g(\omega)$ is an $\tilde{F}$-measurable simple function. Let $g(\omega)$ be a simple function such that $g(\omega)=\left\{a_{i}, F_{i}\right\}(i=1,2, \ldots)$

$$
\begin{aligned}
& \lim _{n \rightarrow \infty} P\left(\sqrt{V}=\frac{1}{n}\left|\sum_{i=1}^{n} X_{i}(\omega)\right| \leqq g(\dot{\omega})\right) \\
= & \lim _{n \rightarrow \infty} \sum_{j=1}^{\infty} P\left(\frac{1}{\gamma}\left|\sum_{i=1}^{n} X_{i}(\omega)\right| \leqq a_{j}, \quad g(\omega)=a_{j}\right) .
\end{aligned}
$$

We can exchange the order of $\lim$ and $\Sigma$ of $(X)$ by the same way as in $3^{\circ}$ of Lemma 1 . We have

$$
\begin{aligned}
& (\mathrm{X})=\sum_{j=1}^{\infty} \lim _{n \rightarrow \infty} P\left(\frac{1}{v}\left|\sum_{i=1}^{n} X_{i}(\omega)\right| \leqq a_{j}, \quad g(\omega)=a_{j}\right) \\
& =\sqrt{ } \frac{1}{2 \pi} \sum_{j=1}^{\infty} P\left(g(\omega)=a_{j}\right) \int_{-(i j}^{a j} e^{-u^{2} / 2} d u=\frac{1}{\frac{2 \pi}{2 \pi}} \int_{\Omega} P(d \omega) \int_{-g(\omega)}^{g(\omega)} e^{-u^{2} / 2} d u .
\end{aligned}
$$

Two measurable functions $g_{1}(\omega)$ and $g_{2}(\omega)$ have the distribution functions $G_{1}(u)$ and $G_{s}(u)$ respectively, and if $G_{1}(u)=G_{s}(u)$ holds for the continuous points, then it is said that $g_{1}(\omega)$ and $g_{3}(\omega)$ have the same distribution function $G_{1}(u)$ (or $G_{2}(u)$ ).

Corollary 1. Let $g_{1}(\omega)$ and $g_{2}(\omega)$ be non-negative $\tilde{F}$-measurable functions having the same distribution function $\bar{G}(u)$. Then

$$
\begin{aligned}
\lim _{n \rightarrow \infty} P & \left(\frac{1}{\sqrt{n}}\left|\sum_{i=1}^{n} X_{i}(\omega)\right| \leqq g_{1}(\omega)\right) \\
& =\lim _{n \rightarrow \infty} P\left(\sqrt{ }=\frac{1}{n}\left|\sum_{i=1}^{n} X_{i}(\omega)\right| \leqq g_{2}(\omega)\right)=\frac{1}{\sqrt{2 \pi}} \int_{0}^{\infty} d \bar{G}(v) \int_{-i}^{v} e^{-u^{2} / 2} d u .
\end{aligned}
$$

Proof. From Theorem 2.

$$
\begin{aligned}
& \lim _{n \rightarrow \infty} P\left(\sqrt{\frac{1}{u}}\left|\sum_{i=1}^{n} X_{i}(\omega)\right| \leqq g_{1}(\omega)\right)=\frac{1}{\sqrt{2 \pi}} \int_{\Omega} P(d \omega) \int_{-g_{1}(\omega)}^{g_{1}(\omega)} e^{-u^{2} / 2} d u \\
& =\frac{1}{V^{\prime}} \int_{0}^{\infty} d \bar{G}(v) \int_{-v}^{v} e^{-u^{2} / 2} d u=\frac{1}{\sqrt{2 \pi}} \int_{\Omega} P(d \omega) \int_{-g_{2}(\omega)}^{g_{2}(\omega)} e^{-u^{2} / 2} d u \\
& =\lim _{n \uparrow \infty} P\left(\sqrt{ }=\frac{1}{\bar{n}}\left|\sum_{i=1}^{n} X_{i}(\omega)\right| \leqq g_{2}(\omega)\right) .
\end{aligned}
$$

Next consider the second generalization.

Theorem 3. Let $N_{n}(\omega)=n N(\omega)+Q_{n}(\omega)$, where $N_{n}(\omega)$ and $N(\omega)$ are $P$-measurable functions which takes non-negative integers, and $Q_{22}(\omega)=O(\sqrt{n})$. If $N(\omega)$ is $\tilde{H}^{\prime}$-measurable, then

$$
\begin{aligned}
\lim _{n \rightarrow \infty} P( & \left.=\frac{1}{n}\left|\sum_{i=0}^{N n(\omega)} X_{i}(\omega)\right| \leqq a\right) \\
& =\sum_{M=0}^{\infty} P(N(\omega)=M) \frac{1}{\sqrt{2 \pi}} \int_{-\alpha M^{-1 / 2}}^{a M^{-1 / 2}} e^{-u^{2} / 2} d u \\
& =\int_{\Omega} P(d \omega) \sqrt{\frac{1}{2 \pi}} \int_{-a N(\omega)^{-1 / 2}}^{a N(\omega)^{-1 / 2}} e^{-u^{2 / 2}} d u .
\end{aligned}
$$


Proof. Let us put $\frac{1}{v^{\prime}} \sum_{i=0}^{N_{22}(\omega)} X(\omega)=\frac{1}{\gamma} \sum_{i=0}^{n n N(\omega)} X_{i}(\omega)+S_{22}(\omega)$. First, we prove that $S_{\imath 2}(\omega)$ converges in probability to 0 . For, $\lim P\left(\left|S_{22}(\omega)\right|>\varepsilon\right)$

$$
\begin{gathered}
=\lim _{n \rightarrow \infty} P\left(\left|S_{n}(\omega)\right|>\varepsilon, \quad \bigcup_{k=0}^{\infty}\left(N(\omega)=M, \bigcup_{k=-\infty}^{\infty} Q_{n}(\omega)=k\right)\right) \\
=\sum_{i=0}^{\infty} \lim _{n \rightarrow \infty} P\left(\left|S_{n}(\omega)\right|>\varepsilon, \quad N(\omega)=M, \bigcup_{k=-\infty}^{\infty} Q_{n}(\omega)=k\right) \\
\quad \leqq \sum_{k=0}^{\infty} \lim _{n \rightarrow \infty} P\left(\frac{1}{\sqrt{n}}\left|\sum^{\prime} X_{i}(\omega)\right|>\varepsilon\right),
\end{gathered}
$$

where $\Sigma^{\prime}$ denotes the summation from $n M$ to $n M+Q_{n}(\omega)$ or from $n M-\left|Q_{n}(\omega)\right|$ to $n M$ according as $Q_{n}(\omega) \geqq 0$ or $Q_{n 2}(\omega)<0$. On the other hand $\left\{X_{i}\right\} i=1,2, \ldots$ satisfies the central limit theorem (I) and $Q_{n}(\omega)=0\left(n^{1 / 2}\right)$ as $n \rightarrow \infty$. Hence

$$
\lim _{n \rightarrow \infty} P\left(v \frac{1}{n}\left|\sum^{\prime} X_{i}(\omega)\right|>\varepsilon\right)=0 \text {. }
$$

So

$$
\begin{aligned}
& \lim _{n \rightarrow \infty} P\left(\sqrt{n}\left|\sum_{i=1}^{N(n)} X_{i}(\omega)\right| \leqq a\right)=\lim _{n \rightarrow \infty} P\left(\frac{1}{\sqrt{n}}\left|\sum_{i=1}^{n N N(\omega)} X_{i}(\omega)\right| \leqq a\right) \\
& =\lim _{n \rightarrow \infty} \sum_{M=0}^{\infty} P\left(\frac{1}{n}\left|\sum_{i=0}^{i n n} X_{i}(\omega)\right| \leqq \alpha, \quad N(\omega)=M\right) \\
& =\lim _{n \rightarrow \infty} \sum_{i=0}^{\infty} P\left(\frac{1}{v^{\prime} n M}\left|\sum_{i=0}^{2 M} X_{i}(\omega)\right| \leqq a M^{-1 / 2}, \quad N(\omega)=M\right) \\
& =\sum_{M=0}^{\infty} \lim P\left(\frac{1}{\gamma M} \sum_{i=0}^{n M} X_{i}(\omega) \mid \leqq a M^{-1 / 2}, \quad N(\omega)=M\right) \\
& =\sum_{M=0}^{\infty} P(N(\omega)=M) \frac{1}{\sqrt{2 \pi}} \int_{-a M^{-1 / 2}}^{a M^{-1 / 2}} e^{-u^{2} / 2} d u \\
& =\frac{1}{\sqrt{2 \pi}} \int_{\Omega} P(d \omega) \int_{-a N(\omega)^{-1 / 2}}^{a N(\omega)^{-1 / 2}} e^{-u^{2 / 2}} d u \text {. }
\end{aligned}
$$

In Theorem 3, when $M=N(\omega)=0, \quad \pm a M^{-1 / 2}$ and $\pm a N(\omega)^{-1 / 2}$ denote $\pm \infty$.

Corollary 2. If $N^{\prime}{ }_{23}(\omega)=n N^{\prime}(\omega)+Q^{\prime}{ }_{n}(\omega)$ and $N^{\prime \prime}{ }_{n 2}(\omega)=n N^{\prime \prime}(\omega)$ $+Q^{\prime \prime}{ }_{n}(\omega)$ satisfy the conditions of $N_{n}(\omega), N(\omega)$ and $Q_{n}(\omega)$ in Theorem 3 , and $N^{\prime}(\omega), N^{\prime \prime}(\omega)$ have the same distribution function $\bar{G}(u)$, then $\quad \lim _{n \rightarrow \infty} P\left(\left.v \frac{1}{n}\right|_{i=0} ^{N_{n}^{\prime}(\omega)} X_{i}(\omega) \mid \leqq a\right)$

$$
=\lim _{n \rightarrow \infty} P\left(v^{\prime} \frac{1}{n}\left|\sum_{i=0}^{N_{n}^{\prime \prime}(\omega)} X_{i}(\omega)\right| \leqq a\right)=\frac{1}{\sqrt{2 n}} \int_{0}^{\infty} d \bar{G}(v) \int_{-a v^{-1 / 2}}^{a v^{-1 / 2}} e^{-u^{2} / 2} d u .
$$

Proof is evident from Theorem 3. 